\title{
Polarization Division Multiplexing of OFDM Radio-over-Fiber Signals in Passive Optical Networks
}

\author{
Maria Morant, Joaquin Pérez, and Roberto Llorente \\ Universitat Politècnica de València, Camino de Vera s/n, 46022 Valencia, Spain \\ Correspondence should be addressed to Maria Morant; mmorant@ntc.upv.es \\ Received 31 July 2013; Accepted 13 January 2014; Published 27 February 2014 \\ Academic Editor: Zoran Ikonic
}

Copyright ( 2014 Maria Morant et al. This is an open access article distributed under the Creative Commons Attribution License, which permits unrestricted use, distribution, and reproduction in any medium, provided the original work is properly cited.

\begin{abstract}
This paper describes the state-of-the-art of polarization multiplexing for optical networks transmission. The use of polarization division multiplexing (PDM) permits to multiply the user capacity and increase the spectral efficiency. Combining PDM and orthogonal frequency division multiplexed (OFDM) modulation allows maximizing the optical transmission capacity. The experimental demonstration of transmitting OFDM signals following ECMA-368 ultrawide band (UWB) standard in radio-overfiber using PDM in passive optical networks is herein reported. The impact of cross-polarization and cochannel crosstalk is evaluated experimentally in a three-user OFDM-UWB subcarrier multiplexed (SCM) configuration per polarization. Each SCM uses up to three OFDM-UWB channels of $200 \mathrm{Mbit} / \mathrm{s}$ each, achieving an aggregated bitrate of $1.2 \mathrm{Gbit} / \mathrm{s}$ with $0.76 \mathrm{bit} / \mathrm{s} / \mathrm{Hz}$ spectral efficiency when using PDM transmission. The experimental results for the polarization-multiplexed SCM indicate that a $4 \mathrm{~dB}$ additional polarization crosstalk interference can be expected compared to a nonpolarization-multiplexed transmission system which translates to $2.4 \mathrm{~dB}$ EVM penalty in the UWB signals. The successful PDM transmission of SCM multiuser OFDM-UWB over a passive optical network of $25 \mathrm{~km}$ standard-single mode fiber (SSMF) reach is demonstrated.
\end{abstract}

\section{Introduction}

The ever-growing transmission capacity demand in optical transmission systems has brought out the necessity of increasing the spectral efficiency employing different transmission techniques. Several approaches have been proposed to date in order to increase the transmission capacity, that is, bitrate by number of users. In particular, the use of efficient modulation formats altogether with advanced multiplexing techniques has been indicated as an interesting approach.

New modulation formats have been reported suitable for providing higher bitrate to the final customer. Especially, orthogonal frequency division multiplexing (OFDM) modulation has been adopted by several wireless standards such as 3GPP long-term evolution (LTE) or IEEE 802.16 worldwide interoperability for microwave access (WiMAX), between others. Also, new transmission technologies based on spreading techniques such as ultrawide band (UWB) use OFDM modulation. These signals can achieve high spectral efficiency when using several hundreds of OFDM subcarriers. Another advantage of the OFDM signals is the capability to correct multipath fading thanks to the cyclic prefix of the OFDM signal. For these reasons, OFDM suitability for optical communications has attracted a lot of attention in the fiber-optic community in the last decade [1].

Optical communication systems are developing rapidly to provide high-bitrate services to the final customer using fiber-to-the-home (FTTH) networks. Moreover, as the use of wireless services employing OFDM modulation is widely extended, radio-over-fiber has been appointed as a rapid and cost-effective solution to facilitate the deployment of integrated access networks and to reach customer premises with high bit rate wireless signals [2]. Using a radio-over-fiber system, we can transport different OFDM-based wireless signals in its native format and regulated frequency band.

However, the ever-growing wireless services demand jointly with the radio spectral scarcity pointed out the necessity of increasing the spectral efficiency, combining new modulation formats with advanced multiplexing techniques.

One optical technique used to improve the efficiency of optical communication systems is polarization division multiplexing (PDM). The use of PDM permits multiplying 

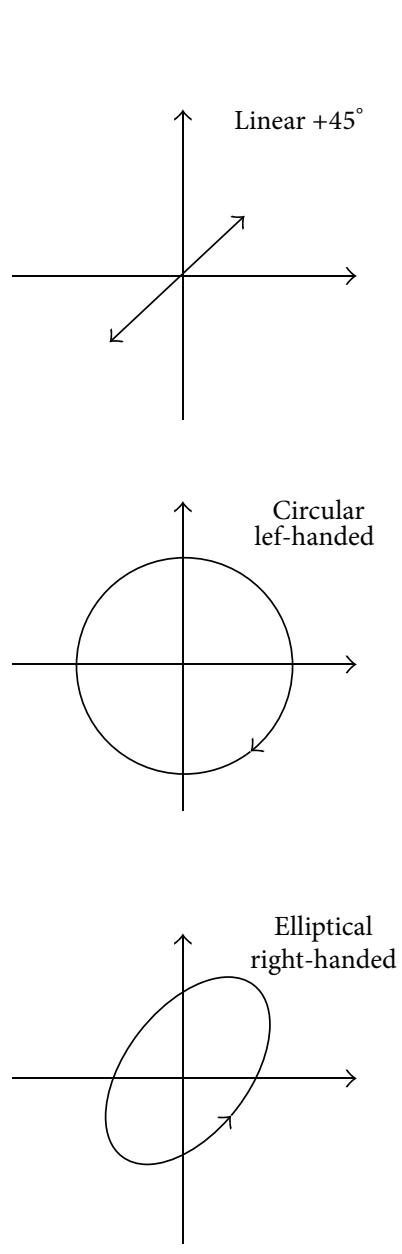

(a)
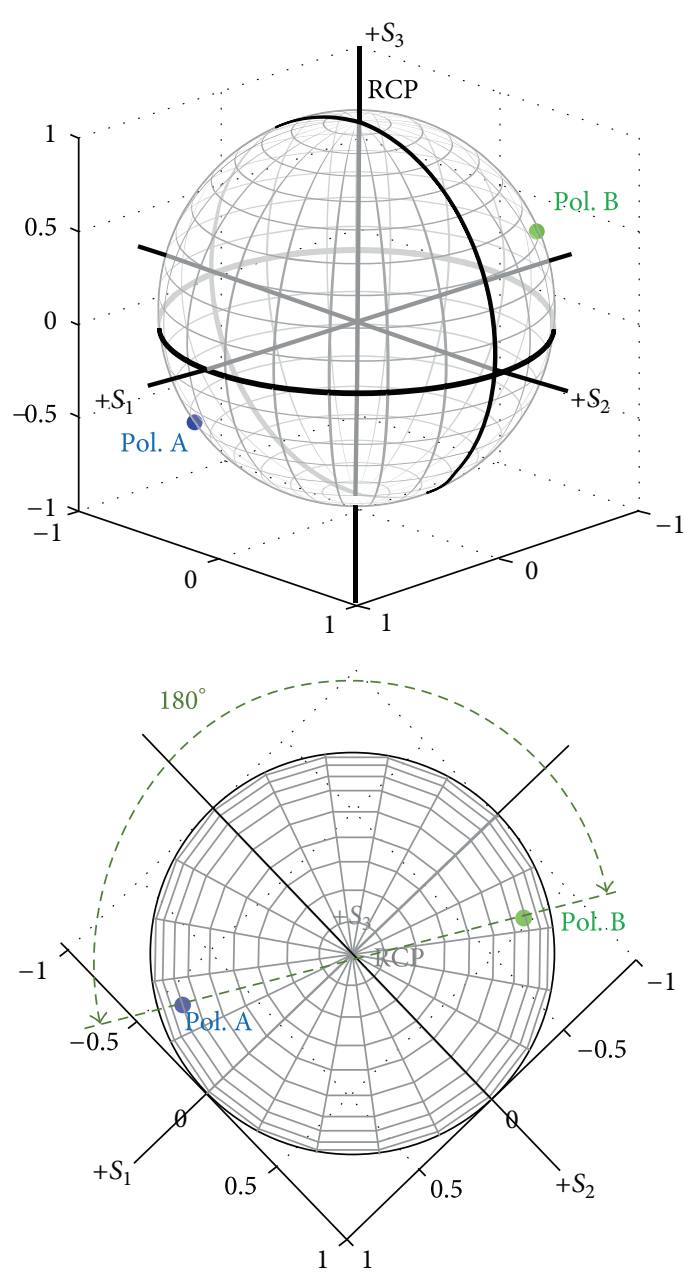

(b)

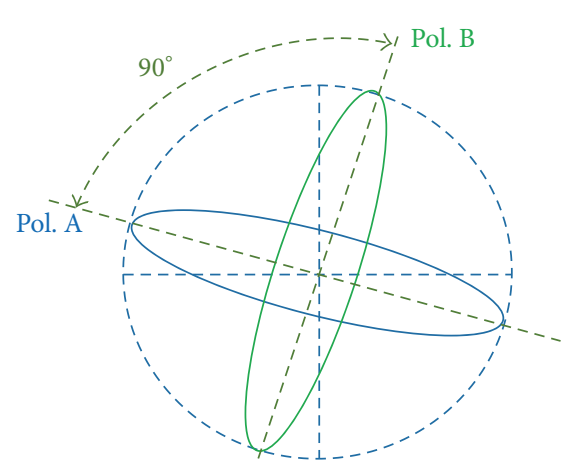

(c)
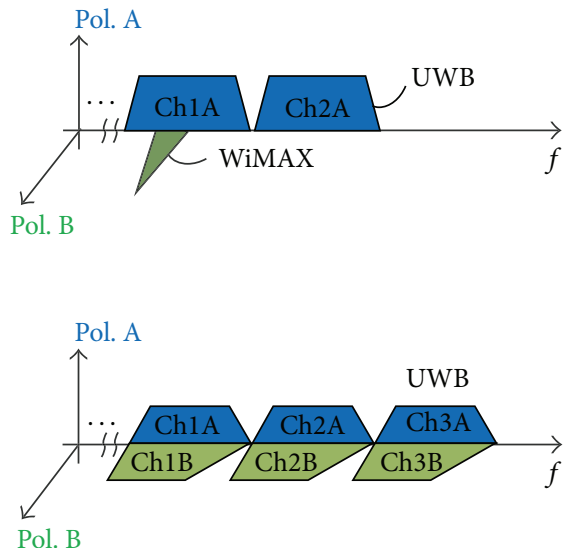

(d)

FIGURE 1: (a) Examples of linear, circular, and elliptical SOP. (b) Orthogonal polarization representation in Poincaré sphere. (c) Example of orthogonal ellipses of polarization. (d) Spectrum allocation examples for PDM transmission systems.

the transmission capacity, as different signals can be transmitted over orthogonal states of polarization of the same light [3].

Another technique used to increase the capacity of optical networks is subcarrier multiplexing (SCM). The SCM method combines several electrical signals at different frequencies to be transmitted over the same optical light. SCM-based passive optical networks (PONs) enable multiple users to share the optical channel and the associated optical components which reduces the overall cost of the network [4]. The use of SCM techniques with OFDM-based signals has been facilitated in the last years by the market availability of cost-effective electrooptic modulators with large frequency range and good linearity [5]. The combination of the PDM and SCM techniques opens up the opportunity of maximizing the capacity of optical networks. In order to combine the advantages of the different techniques; this paper reports a PDM radio-over-fiber system for the transmission of SCM OFDM-based signals in passive optical networks.

This paper is structured as follows. In Section 2, an overview of the progress of PDM techniques through the last years is included. In Section 3, the performance of a radio-over-fiber system transmitting full-standard OFDM modulated signals combining PDM and SCM is evaluated experimentally. The cross-polarization interference when using SCM transmission in a PDM radio-over-fiber system is studied in this work analyzing different OFDM-UWB channels. The maximum PON reach that can be achieved using the combination of PDM and SCM OFDM radio-overfiber transmission is also measured experimentally. Finally, in Section 4, the main conclusions are highlighted.

\section{Optical Polarization Division Multiplexing Overview}

Optical polarization division multiplexing is based on transmitting independently modulated signals over orthogonal polarizations of the same optical wavelength. In optics, the state of optical polarization (SOP) is defined as the shape traced out by the electric-field vector of the transmitted light in a fixed plane. Figure 1(a) shows some examples of SOP, including linear, circular, and elliptical polarization states. 
Linear polarization is obtained when the direction of the electric vector is constant (the electric-field vectors along with $E_{x}$ and $E_{y}$ directions are in phase). When $E_{x}$ and $E_{y}$ are exactly ninety degrees out of phase, we have circular polarization. There are right-handed or left-handed circular/elliptical polarizations, depending on which direction the electric-field vector rotates, that is, if the electric-field vector is seen rotating clockwise or counterclockwise.

Figure 1(b) shows an example of two orthogonal SOP represented in the Poincare sphere. The polarization state is defined using the Stokes parameters that can be represented in the Poincare sphere over the three-dimensional vector of Cartesian coordinates called $S_{1}, S_{2}$, and $S_{3}$. The right circular polarization pole (RCP) is included in the sphere for reference. In the example presented in Figures 1(b) and 1(c), the polarization states were measured with Optellios PS2300 optical polarization analyzer. As it is described in Figure 1(b), two polarizations are orthogonal if they are separated by $180^{\circ}$ in the Poincaré sphere. Figure 1(c) shows the polarization ellipses for each polarization. In the representation of polarization ellipses, two states are orthogonal if they have a $90^{\circ}$ phase shift (as sine and cosine).

The polarization multiplexing method can be explained from the similarity with the techniques used in microwave communications. In wireless links, the user bandwidth can be improved if two orthogonally polarized radio frequency signals are transmitted. At the receiver, two antennas with different polarization and orientation are used to discriminate each of the signals. The same occurs in optical systems: at the receiver, the two orthogonal states of polarization are detected, obtaining each of the modulated signals independently. If the orthogonality is maintained through the optical system, at the receiver, each of the modulated signals can be recovered. But SOP orthogonality is degraded due to the propagation in optical networks [6] due to stress in the glass fiber (bending and twisting), moving the fiber, or even ambient temperature changes. For this reason, it is important to evaluate if the crosstalk due to cross-polarization interference is limiting the performance at the receiver.

Several techniques have been proposed to mitigate the interference coming from cross-polarization. One technique is based on frequency interleaving of the adjacent channels that are orthogonally polarized [7]. Polarization interleave multiplexing systems have achieved experimentally up to $1.6 \mathrm{bit} / \mathrm{s} / \mathrm{Hz}$ spectrum efficiency as demonstrated by Sotobayashi et al. [8].

The main impairments suffered by optical PDM systems are due to cross-phase modulation (XPM) [9] and polarization-mode dispersion (PMD) [10]. Nelson et al. [11] assessed the impairments caused by first-order PMD in the fiber using a non-return-to-zero (NRZ) polarization-multiplexed system with $40 \mathrm{~Gb} / \mathrm{s}$ per channel and $0.8 \mathrm{bit} / \mathrm{s} / \mathrm{Hz}$ spectral efficiency. The measured system penalty of $1 \mathrm{~dB}$ in instantaneous differential group delay (DGD) pointed out that polarizationmultiplexed systems are five times more sensitive to PMD compared with nonpolarization-multiplexed systems due to crosstalk [11]. However, a $121.9 \mathrm{~Gb} / \mathrm{s}$ polarization-multiplexed transmission using coherent detection was demonstrated by Jansen et al. [12] with 4 OFDM channels at $2 \mathrm{bit} / \mathrm{s} / \mathrm{Hz}$ spectral efficiency over $1000 \mathrm{~km}$ of standard-single mode fiber (SSMF).

The receiver of PDM-OFDM system can be seen as a multiple input multiple output (MIMO) system $2 \times 2$. Thus, digital signal processing (DSP) algorithm based on MIMO processing can be used for improving the optical transmission, as proposed in the literature for wireless communications [13]. Recently, PDM systems have been demonstrated as a viable solution for fully standardized wireless MIMO provision using radio-over-fiber polarization-multiplexed long-reach optical transmission [14]. Two different optical detection approaches can be implemented: direct or coherent detection. For simplicity of the receiver, in this paper, we will focus on direct detection, but the network reach could be further extended using coherent detection or adding MIMO digital processing. For example, a PON architecture of $20 \mathrm{~km}$ of SSMF was demonstrated with a $40 \mathrm{~Gb} / \mathrm{s}$ PDM-OFDM transmission including MIMO digital signal processing at the receiver [15].

\section{PDM OFDM-UWB Radio-over-Fiber Transmission System}

As it was introduced previously, OFDM has been appointed as an effective modulation scheme to increase the user bitrate and spectral efficiency. Although the OFDM signal needs additional overheads due to the cyclic prefix, the training symbols for channel estimation, and pilot subcarriers for phase noise compensation, the performance of OFDM signals is more robust to multipath fading and to the impairments caused by chromatic dispersion or PMD. For these reasons, using OFDM signals can increase the efficiency and the flexibility of access optical networks while reducing implementation cost. However, due to the limited technology of commercial digital-to-analog converters (DAC) [15], in order to obtain very high bitrates, the combination of PDM and OFDM systems is an interesting solution.

As OFDM signals are currently used by most of the useroriented wireless standards (such as DVB-T, WiMAX, LTE, or UWB), we propose to combine PDM distribution in a radioover-fiber system transmitting full-standard OFDM signals in its native format. The OFDM signals is transmitted in the corresponding regulated frequency band, so no upconversion or frequency shift is needed at the receiver, which simplifies the optical network terminator at customer premises [16] and, in consequence, reduces the cost for both the final user and the operator. Moreover, the radio-over-fiber systems are transparent to the signals that are transmitted through the network, which enables including new signals to the network as soon as they appear in the market [17]. In this case, we selected OFDM-UWB signals for the evaluation of the PDM transmission in passive optical networks. UWB radio operates in a large frequency range (i.e., from 3.1 to $10.6 \mathrm{GHz}$ ) and emits very low power spectral densities $(-41.3 \mathrm{dBm} / \mathrm{MHz})$ to minimize the interference over other radio services [18]. In ECMA standard 368, the UWB band is divided in 12 channels of $528 \mathrm{MHz}$ bandwidth each for OFDM signals with 128 subcarriers [19]. These are also called multiband OFDM signals. The growing interest of UWB technology focuses 


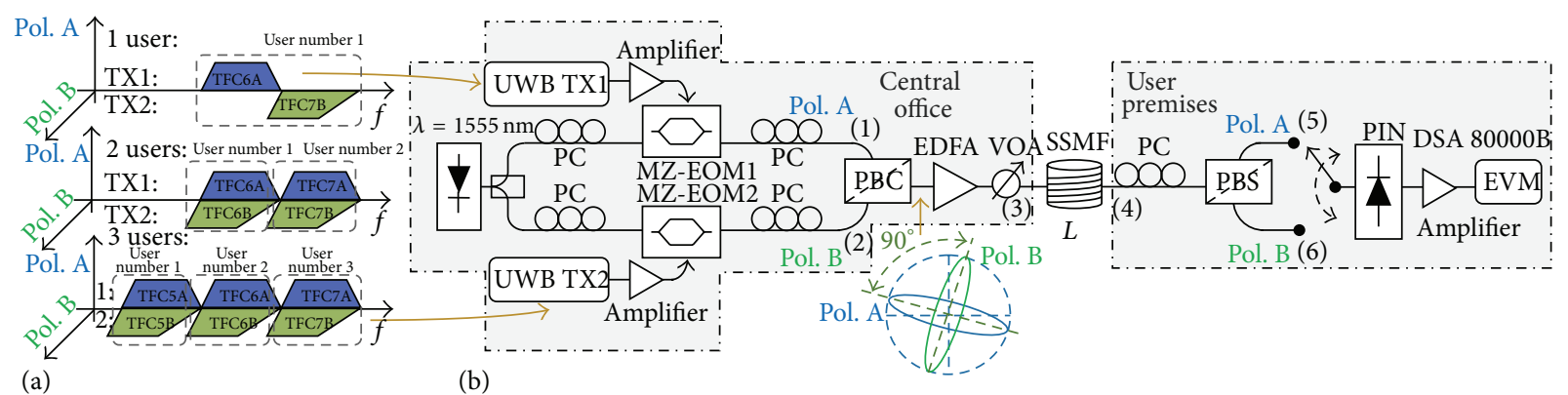

FIGURE 2: (a) SCM-UWB multiuser configurations using two polarizations. (b) Experimental setup for SCM-UWB transmission with polarization division multiplexing.

on short range communications in indoor environments like wireless personal area networks (WPAN) working with low-cost receivers in UWB enabled commercial available devices such as televisions or laptops. The radio-over-fiber transmission of UWB signals for audio/video distribution was first proposed in [20] analyzing the maximum reach on a single light in FTTH networks including optical amplification sections when necessary.

The first demonstration of PDM distribution of OFDMUWB signals was reported in [21]. As it was commented previously, using a radio-over-fiber system, we are able to transmit different standard OFDM signals transparently to the optical network. The use of polarization multiplexing for joint UWB and WiMAX transmission was proposed in [22], demonstrating the transmission of different OFDM services on each of the orthogonal optical polarizations. As it is represented in Figure 1(d), one polarization can be used for transmitting WiMAX signals and the orthogonal polarization for UWB broadcasting.

Polarization multiplexed capacity can be further exploited if large frequency data is transmitted in both polarizations, and even more if we employ SCM for the transmission of multiple UWB channels [23]. So, in this paper, we evaluate experimentally the performance of a PDM passive optical network transmitting several OFDM-UWB channels in a SCM configuration in both polarizations. As it can be seen in Figure 2(a), we can transmit up three different UWB channels (full UWB band number 1) in polarization $A$ and other three UWB channels in polarization B with different SCM configurations.

3.1. Experimental Setup. Figure 2(b) shows the experimental setup where the central office transmitter generates a single wavelength at $1555 \mathrm{~nm}$. The UWB user data is generated by commercial low-cost transmitters from Wisair following ECMA-268 standard and WiMedia specification. Different SCM multiuser configurations are used in the experiments as depicted in Figure 2(a), based on different ECMA-368 time frequency codes (TFC). We consider the first UWB band located from 3.1 to $4.8 \mathrm{GHz}$ (comprising three UWB channels) which is used by devices currently available in the market. Employing PDM with two orthogonal SOP, we are able to multiply by two the capacity for each user and, in consequence, the spectral efficiency is doubled. Using the first
UWB band, we can transmit three UWB channels in each polarization. So, with three UWB channels at $200 \mathrm{Mbit} / \mathrm{s}$ in $528 \mathrm{MHz}$ each per polarization, we obtain a spectral efficiency of $0.76 \mathrm{bit} / \mathrm{s} / \mathrm{Hz}$. It is important to note that this kind of simultaneous TFC transmission could not be possible without using polarization multiplexing, as the receiver would not be able to separate both transmissions using conventional wireless transmission.

At the transmitter, the multiuser UWB signal is amplified (with a wideband amplifier, Picosecond 5865) and modulated at quadrature bias point on the two orthogonal polarizations of the same light by two Mach-Zehnder electrooptical modulators (MZ-EOM). The modulated signals at points (1) and (2) in Figure 2(b) are adjusted with polarization controllers (PC) to orthogonal SOP. These polarizations (labelled as polarization $\mathrm{A}$ and polarization $\mathrm{B}$ ) were measured with an optical polarization analyzer (Optellios PS2300) and represented in the Poincaré sphere in Figure 1(b). The Stokes parameters are measured as $\left[S_{1}, S_{2}, S_{3}\right]=[0.77,-0.46,-0.42]$ for polarization $A$ and $\left[S_{1}, S_{2}, S_{3}\right]=[-0.75,0.48,0.43]$ for polarization $\mathrm{B}$. The degree of polarization (DOP) parameter is $99.06 \%$ for Pol. A and $98.98 \%$ for Pol. B (considering that a totally polarized light has DOP $=1$ ). The ellipticity of both polarizations is 0.22 . As it can be observed in the figure, the polarization ellipses have $90^{\circ}$ difference in its orientation, thus confirming the polarization orthogonality.

Both orthogonal polarizations are combined by a polarization beam combiner (PBC). The PDM-UWB signal is boosted by Erbium doped fiber amplifier (EDFA Amonics 30-B-FA) at the output of the central office. This amplifier is used for evaluation purposes in order to control the power launched into the fiber. Different SSMF reaches expected in PON access [2] are evaluated as follows: $L=5,10,25$, and $50 \mathrm{~km}$. The PDM-UWB signal is received at point (4) in Figure 2(b), where the two polarizations are split, directly photodetected, filtered, and amplified. No demodulation or upconversion stages are required using this technique, thus simplifying the receiver.

In the experiments, a commercial polarization controller is used to adjust the polarization arriving to the polarization beam splitter (PBS) located at customer premises. In an onthe-field deployment, this polarization controller would be automatic [24]. Also, adaptive polarization can be used to remove the tracking at the receiver as it was proposed in 


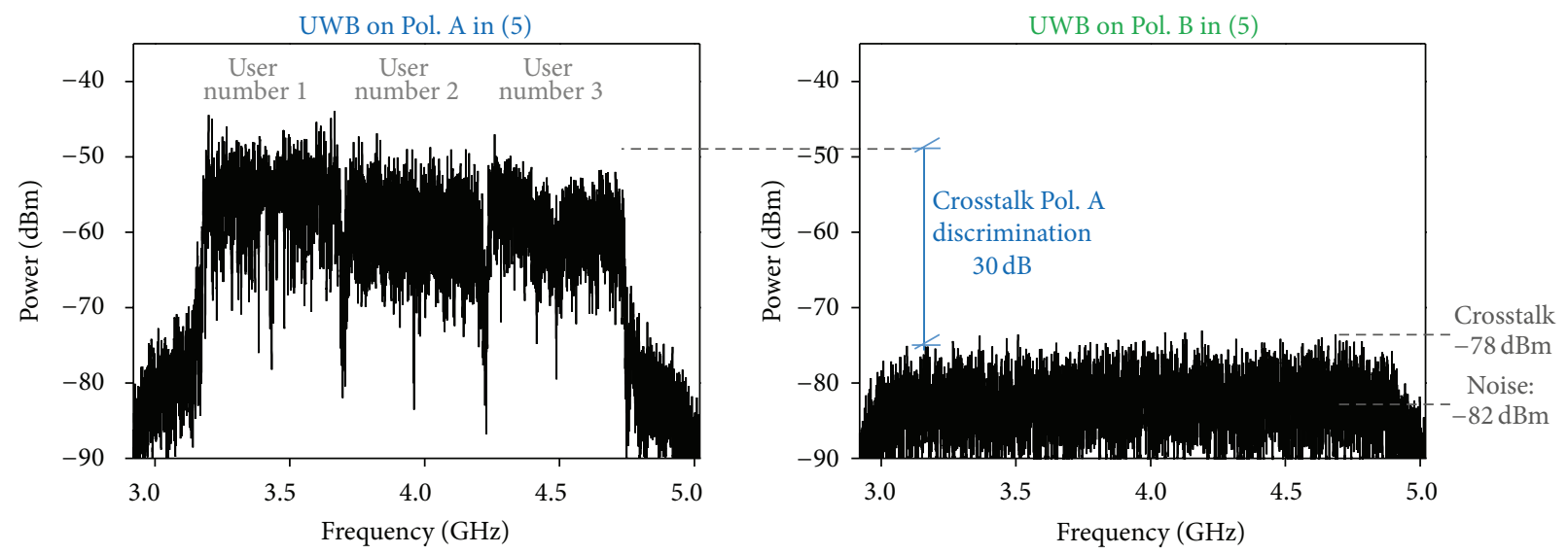

(a)
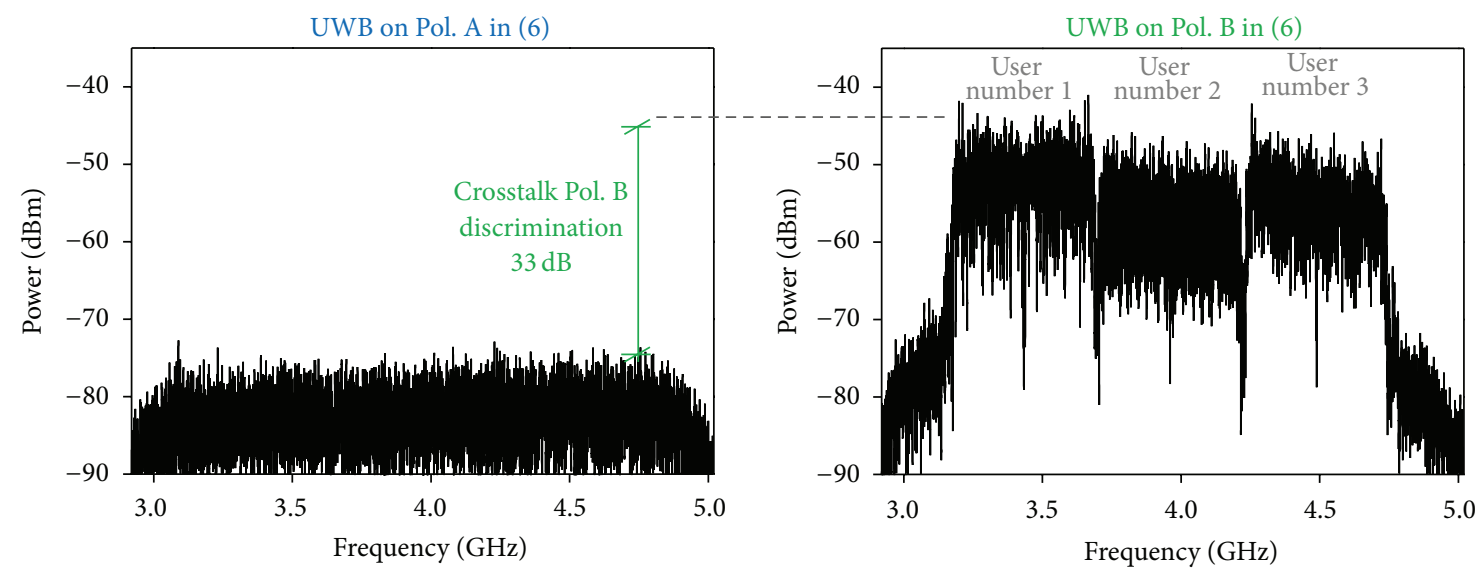

(b)

Figure 3: Cross-polarization residual measurements after $10 \mathrm{~km}$ of SSMF with RF spectrum at RBW $=1 \mathrm{MHz}$ for both polarizations at (a) point (5) and (b) point (6) of Figure 2(b).

[25]. Different optical PDM receiver architectures that can be used to eliminate the necessity of conventional polarization tracking are presented in [26] for both intensity modulation direct detection and coherent receivers. In these experiments, each polarization is detected with a PIN photodetector with $0.7 \mathrm{~A} / \mathrm{W}$ responsivity. The quality of each OFDM-UWB channel is measured sampling the signal with a digital signal analyzer (Agilent DSA80000B) and demodulating the OFDM signal to evaluate the error vector magnitude (EVM). The values of EVM are compared with the threshold provided by ECMA-368 standard of OFDM-UWB signals working at $200 \mathrm{Mbit} / \mathrm{s}$ of $-14.5 \mathrm{~dB}$ [19] to ensure correct operation.

3.2. Evaluation of Cross-Polarization Interference. The residual polarization crosstalk has been measured for both polarization paths. The cross-polarization crosstalk is obtained measuring the received electrical spectrum when the UWB modulated signal is only travelling over one of the polarizations.

Figure 3(a) shows the corresponding spectrum at the receiver measured at point (5) in Figure 2(b) with $L=10 \mathrm{~km}$ when a three-user UWB communications are being provided in Pol. A. The received spectrum is also measured at the same
PBS output-point (5) in Figure 2(b) for $L=10 \mathrm{~km}$ when the UWB signal is transmitted over Pol. B (with Pol. A off). It can be observed that the cross-polarization discrimination is of $30 \mathrm{~dB}$ for Pol. A. The crosstalk produced by Pol. A is measured in $-78 \mathrm{dBm}$ over a $-82 \mathrm{dBm}$ noise floor. This $4 \mathrm{~dB}$ crosstalk will interfere and affect the quality of the received signal. The same is done in Figure 2(b) for Pol. B (point (6)) obtaining a higher polarization discrimination of $33 \mathrm{~dB}$ due to the fabrication characteristics of the PBS. The crosstalk produced by Pol. B is also of $4 \mathrm{~dB}$ over the noise floor.

3.3. Evaluation of SCM Multichannel Crosstalk. Taking into account the cross-polarization over the received signal, the effect of channel aggregation and interchannel distortion is evaluated when multiple OFDM channels are transmitted simultaneously 6 in a PDM system. Figure 4 shows the EVM of the received PDM-UWB signal for each multiuser SCM configuration at different fiber lengths $L$ employing the experimental setup shown in Figure 2(b). The ECMA-368 EVM limit of $-14.5 \mathrm{~dB}$ [19] is represented as a dashed line.

For short PON distances $(L=5 \mathrm{~km})$, we can observe that when passing from no polarization multiplexing to one user PDM, the distortion is only $0.35 \mathrm{~dB}$ in the UWB channel 


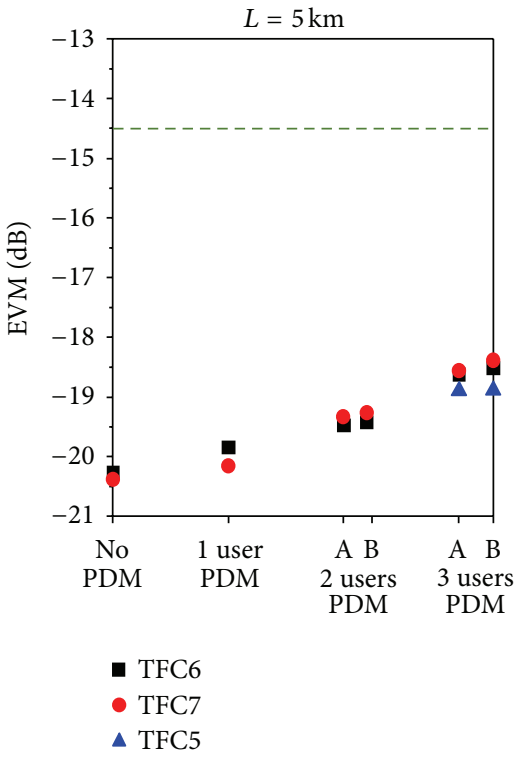

(a)

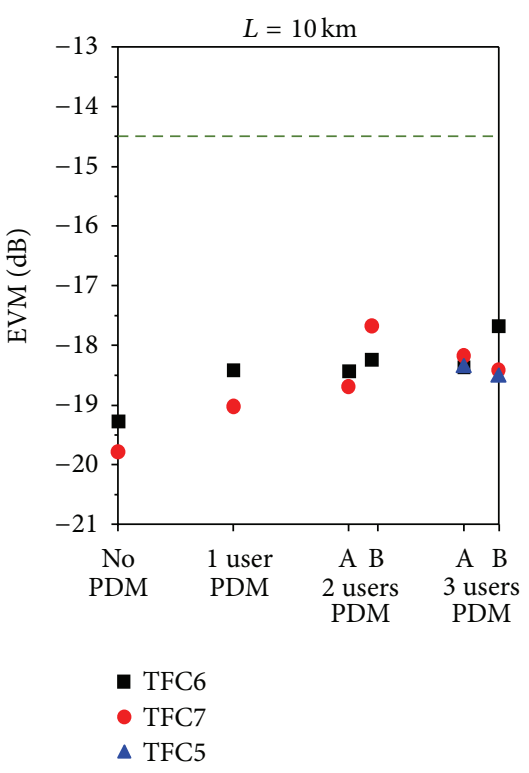

(b)

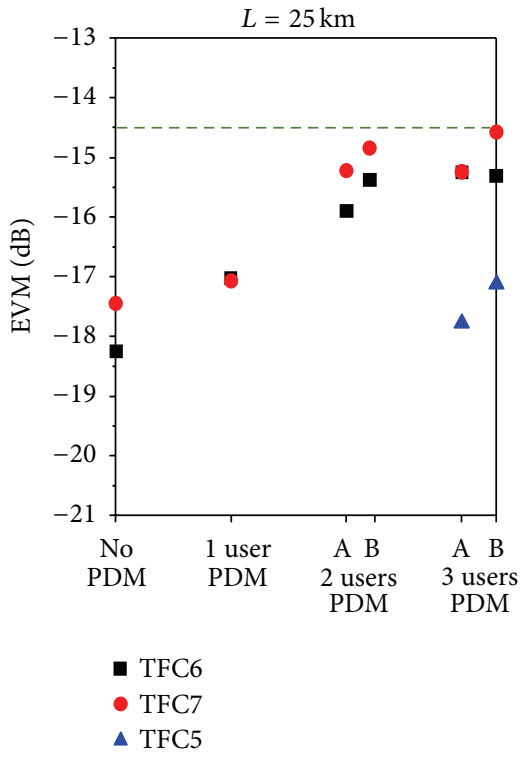

(c)

FIGURE 4: PDM-UWB EVM performance of each UWB channel in different SCM multiuser configurations versus PON reach: (a) $L=5 \mathrm{~km}$, (b) $L=10 \mathrm{~km}$, and (c) $L=25 \mathrm{~km}$.

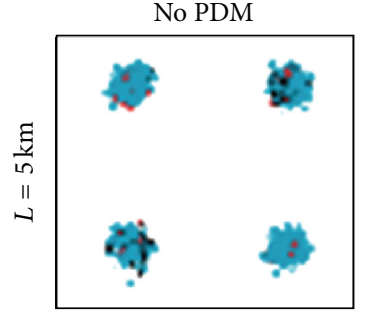

$\mathrm{EVM}=-20.27 \mathrm{~dB}$

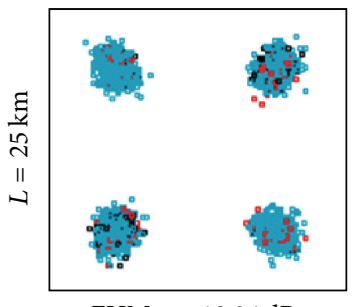

$\mathrm{EVM}=-18.24 \mathrm{~dB}$

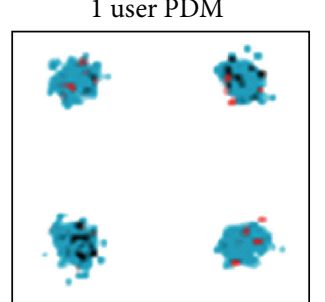

$\mathrm{EVM}=-19.85 \mathrm{~dB}$

(a)

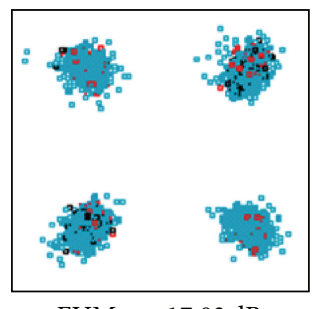

$\mathrm{EVM}=-17.02 \mathrm{~dB}$

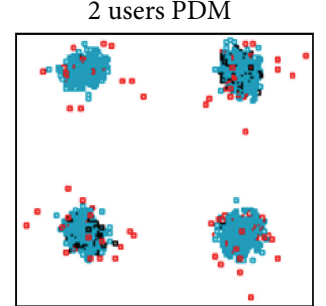

$\mathrm{EVM}=-19.43 \mathrm{~dB}$

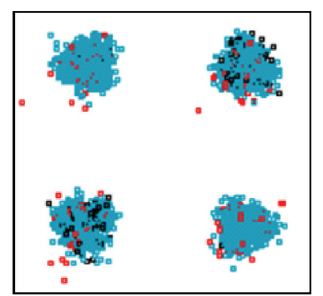

$\mathrm{EVM}=-15.89 \mathrm{~dB}$

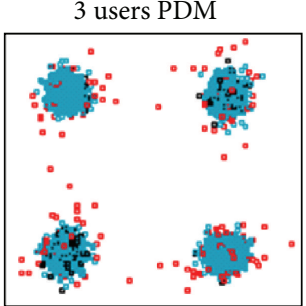

$\mathrm{EVM}=-18.52 \mathrm{~dB}$

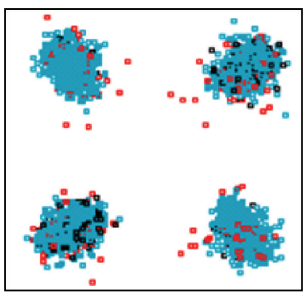

$\mathrm{EVM}=-15.24 \mathrm{~dB}$

(b)

Figure 5: Constellations of the center channel TFC6 with different SCM multiuser configurations using or not PDM for (a) $L=5 \mathrm{~km}$ and (b) $L=25 \mathrm{~km}$. $24 \mathrm{UWB}$ symbols represented the following: blue dots indicate data symbols and red dots correspond to pilot and guard symbols.

working at TFC6. When adding a second user (cochannel interference), the EVM is $0.42 \mathrm{~dB}$ worse than a single user with different TFC. Finally, transmitting the three-user UWB channels, the EVM in TFC6 is $-18.52 \mathrm{~dB}(0.91 \mathrm{~dB}$ worse than two users with PDM). For longer distances $(L=$ $25 \mathrm{~km}$ ), the EVM penalty is of $1.2 \mathrm{~dB}$ from no polarization to single user PDM and of $3 \mathrm{~dB}$ to three users using PDM. Symbol degradation can be seen in the quadrature phase shift keying (QPSK) constellations represented in Figure 5.

3.4. Evaluation of Maximum Reach in PON. Once the effect of multiuser transmission is evaluated, the SCM-UWB performance after $L \mathrm{~km}$ repeater-less optical transmission in PDM networks is studied using different launch power levels. The experimental setup was shown in Figure 2(b), where a 


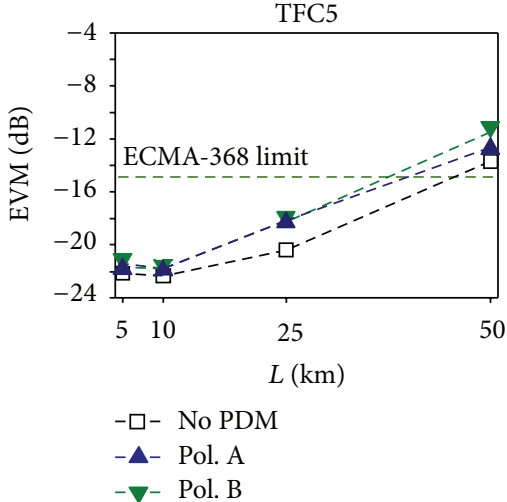

(a)

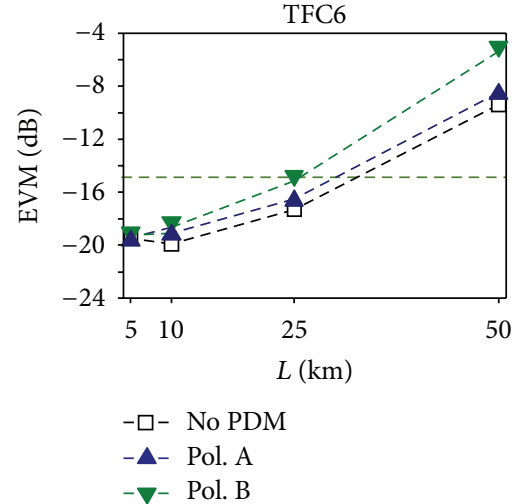

(b)

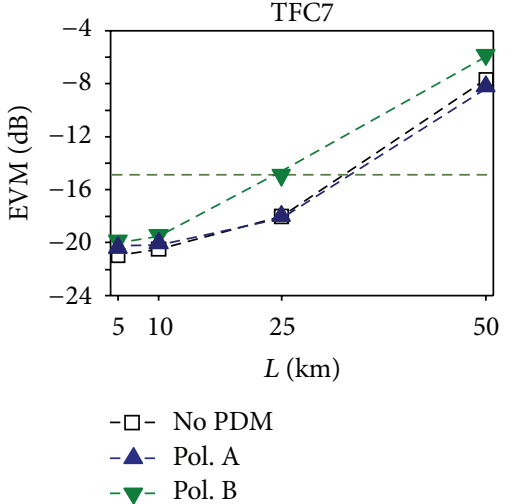

(c)

FIGURE 6: EVM performance using PDM with $7 \mathrm{dBm}$ optical launch power versus using fiber reach $(L)$ for (a) user number 1 TFC5, (b) user number 2 TFC6, and (c) user number 3 TFC7.

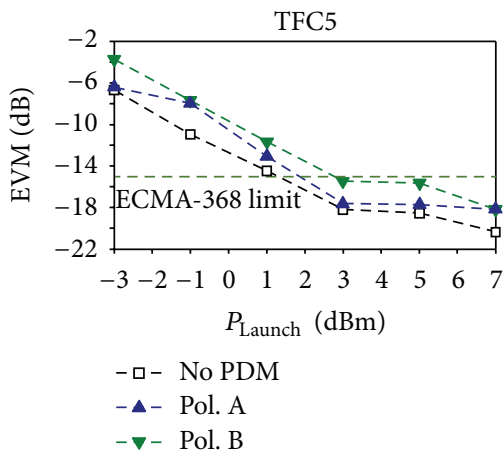

(a)

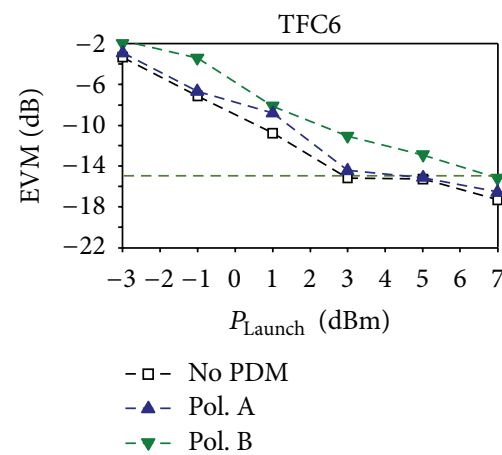

(b)

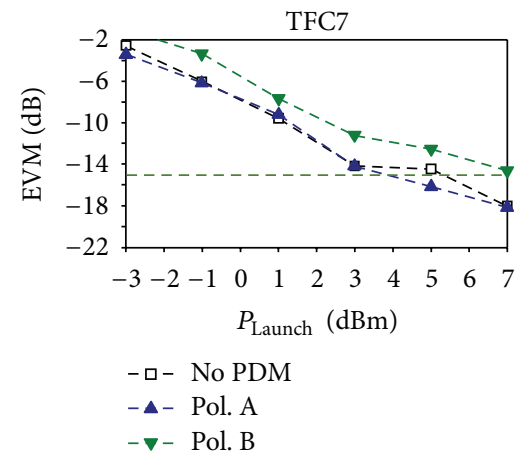

(c)

FIGURE 7: EVM performance using PDM measured after $25 \mathrm{~km}$ SSMF for (a) user number 1 TFC5, (b) user number 2 TFC6, and (c) user number 3 TFC7.

variable optical attenuator (VOA) at point (3) is used to change the optical power level launched from the central office (from -3 to $7 \mathrm{dBm}$ ). The EDFA pump level was kept fixed for all measurements in order to keep the same noise conditions. In this case, the three first UWB channels are transmitted (3 users configuration represented in Figure 2(a)), each one working at $200 \mathrm{Mbit} / \mathrm{s}$.

In order to evaluate the penalty induced by the proposed PDM technique for OFDM-UWB radio-over-fiber distribution, the UWB performance using single polarization is evaluated measuring only one path separately, for example, the signal from point (1) to point (5) in Figure 2(b). The opened blank symbols represent single polarization transmission (No PDM). These results are compared with the performance measured using PDM. The experimental results are shown in Figure 6 for different PON distances from $5 \mathrm{~km}$ to $50 \mathrm{~km}$ of SSMF.

The results indicate that the SCM-UWB multiuser signal transmitted on both polarizations achieve successful communication (EVM under $-14.5 \mathrm{~dB}$ [19]) for each user/TFC over PON distances up to $25 \mathrm{~km}$ of SSMF. A $50 \mathrm{~km}$ network could be reached for the lowest frequency UWB channel if extra error correction is applied. For higher frequency,
UWB channels dispersion compensation [27] or intermediate optical amplification [16] would be needed.

The performance with the launch power is represented in Figure 7 for $L=25 \mathrm{~km}$. Comparing the results obtained with PDM to the ones with single polarization (No PDM), it can be observed that the interference between orthogonal polarizations induces a maximum penalty of $5.5 \mathrm{~dB}$ when the launch power level is below $0 \mathrm{dBm}$, and less than $2.4 \mathrm{~dB}$, if it is above $3 \mathrm{dBm}$. So, according to the experimental results, the reach of the proposed PDM system is estimated in $25 \mathrm{~km}$ using a launch power level from the central office of $5 \mathrm{dBm}$ which is adequate for typical PON networks deployments [4].

\section{Conclusion}

This paper reviews the progress of polarization division multiplexing techniques applied to optical networks communications and in particular to radio-over-fiber transmission. PDM is based on transmitting independently modulated signals over orthogonal polarizations of the same optical wavelength which permits multiplying the bitrate and the spectral efficiency transmission. As the polarization changes with the fiber propagation, we evaluated the 
cross-polarization interference in radio-over-fiber systems with the transmission of standard OFDM-based UWB signals. Also, the cochannel crosstalk was evaluated experimentally combining PDM with a three-user SCM configuration. Three UWB channels working each at $200 \mathrm{Mbit} / \mathrm{s}$ were transmitted in a PDM radio-over-fiber system, achieving an aggregated bitrate of $1.2 \mathrm{Gbit} / \mathrm{s}$ with $0.76 \mathrm{bit} / \mathrm{s} / \mathrm{Hz}$ spectral efficiency.

The experimental results for the polarization-multiplexed SCM system indicate that a $4 \mathrm{~dB}$ additional polarization crosstalk interference can be expected compared to a nonpolarization-multiplexed transmission system. This crosstalk interference is translated to $2.4 \mathrm{~dB}$ EVM penalty in the quality of the OFDM-UWB signals.

A successful PDM-UWB transmission was demonstrated for PON of $25 \mathrm{~km} \mathrm{SSMF} \mathrm{for} \mathrm{launch} \mathrm{power} \mathrm{levels}$ of $5 \mathrm{dBm}$ from the central office achieving a better EVM than -14.5 dB ECMA-368 threshold in all three SCM UWB user's channels. This transmission is done without using any impairment compensation technique or inline regeneration stages between the central office and user premises. Further improvement could be achieved using impairment compensation, MIMO digital signal processing at the receiver, or coherent detection.

\section{Conflict of Interests}

The authors declare that there is no conflict of interests regarding the publication of this paper.

\section{Acknowledgments}

This work has been supported by Spain National Plan Project MODAL "Few-mode propagation technology in single mode fibre" (TEC2012-38558-C02-01). UPV Project MUMOX "Optical modal multiplexing for high-performance network interconnection" (PAID-05-12 SP20120821) is also acknowledged. M. Morant's work is supported by Generalitat Valenciana VALi+D Postdoc Program. J. Perez's work is supported by Spanish MINECO Juan de la Cierva Fellowship JCI-2012-14805.

\section{References}

[1] I. Morita, "Optical OFDM for high-speed transmission," in Proceedings of the 14th OptoElectronics and Communications Conference (OECC '09), July 2009.

[2] T. Koonen, M. García Larrodé, P. Urban et al., "Fibre-based versatile broadband access and in-building networks," in Proceedings of the IET Workshop from Access to Metro, December 2007.

[3] M. I. Hayee, M. C. Cardakli, A. B. Sahin, and A. E. Willner, "Doubling of bandwidth utilization using two orthogonal polarizations and power unbalancing in a polarization-divisionmultiplexing scheme," IEEE Photonics Technology Letters, vol. 13, no. 8, pp. 881-883, 2001.

[4] L. G. Kazovsky, W.-T. Shaw, D. Gutierrez, N. Cheng, and S.W. Wong, "Next-generation optical access networks," IEEE/OSA
Journal of Lightwave Technology, vol. 25, no. 11, pp. 3428-3442, 2007.

[5] M. Morant, R. Llorente, J. Hauden, T. Quinlan, A. Mottet, and S. Walker, "Dual-drive $\mathrm{LiNbO}_{3}$ interferometric Mach-Zehnder architecture with extended linear regime for high peak-toaverage OFDM-based communication systems," Optics Express, vol. 19, no. 26, pp. 197-202, 2011.

[6] L. J. Cimini, I. M. I. Habbab, R. K. John, and A. A. M. Saleh, "Preservation of polarization orthogonality through a linear optical system," Electronics Letters, vol. 23, no. 25, pp. 1365-1366, 1987.

[7] T. Ito, T. Ono, Y. Yano et al., "Feasibility study on over $1 \mathrm{bit} / \mathrm{s} / \mathrm{Hz}$ high spectral efficiency WDM with optical duobinary coding and polarization interleave multiplexing," in Proceedings of the Optical Fiber Communication Conference (OFC '97), pp. 43-44, February 1997.

[8] H. Sotobayashi, W. Chujo, and K.-I. Kitayama, "1.6 bit/s/Hz, 6.4 Tbit/s OCDM/WDM $(4 \mathrm{OCDM} \times 40 \mathrm{WDM} \times 40 \mathrm{Gbit} / \mathrm{s})$ transmission experiment," in Proceedings of the 27th European Conference on Optical Communication (ECOC '01), vol. 6, pp. 6-7, 2001.

[9] B. C. Collings and L. Boivin, "Nonlinear polarization evolution induced by cross-phase modulation and its impact on transmission systems," IEEE Photonics Technology Letters, vol. 12, no. 11, pp. 1582-1584, 2000.

[10] J. P. Gordon and H. Kogelnik, "PMD fundamentals: Polarization mode dispersion in optical fibers," Proceedings of the National Academy of Sciences of the United States of America, vol. 97, no. 9, pp. 4541-4550, 2000.

[11] L. E. Nelson, T. N. Nielsen, and H. Kogelnik, "Observation of PMD-induced coherent crosstalk in polarization-multiplexed transmission," IEEE Photonics Technology Letters, vol. 13, no. 7, pp. 378-390, 2001.

[12] S. L. Jansen, I. Morita, and H. Tanaka, " $10 \times 121.9-\mathrm{Gb} / \mathrm{s}$ PDMOFDM transmission with $2-\mathrm{b} / \mathrm{s} / \mathrm{Hz}$ spectral efficiency over $1,000 \mathrm{~km}$ of SSMF," in Proceedings of the Optical Fiber Communication Conference and Exposition/National Fiber Optic Engineers Conference (OFC/NFOEC '08), PDP2, February 2008.

[13] D. Gesbert, M. Shafi, D.-S. Shiu, P. J. Smith, and A. Naguib, "From theory to practice: an overview of MIMO space-time coded wireless systems," IEEE Journal on Selected Areas in Communications, vol. 21, no. 4, pp. 281-282, 2003.

[14] M. Morant, R. Llorente, and J. Prat, "LTE advanced carrier aggregation supporting fully standard 3GPP MIMO by optical polarization multiplexing," in Proceedings of the Optical Fiber Communication Conference and Exposition/National Fiber Optic Engineers Conference (OFC/NFOEC '14), paper M3D.2, March 2014.

[15] D. Qian, N. Cvijetic, J. Hu, and T. Wang, "40-Gb/s MIMOOFDM-PON using polarization multiplexing and directdetection," in Proceedings of Optical Fiber Communication Conference and Exposition/National Fiber Optic Engineers Conference (OFC/NFOEC '09), paper OMV3, March.

[16] M. Morant, J. Perez, R. Llorente, and J. Marti, "Combined analysis of OFDM-UWB transmission in hybrid wireless-optical access networks," IEEE Photonics Technology Letters, vol. 21, no. 19, pp. 1378-1380, 2009.

[17] M. Morant, T. Quinlan, S. Walker, and R. Llorente, "'Real world" FTTH optical-to-radio interface performance for bidirectional multi-format OFDM wireless signal transmission," in Proceedings of the Optical Fiber Communication Conference 
and Exposition and the National Fiber Optic Engineers Conference (OFC/NFOEC '11), paper NTuB6, March 2011.

[18] FCC 02-48, Revision of Part 15 of the Commission's Rules Regarding Ultra-Wideband Transmission Systems, FCC, 2002.

[19] ECMA-368 International Standard, High Rate Ultra Wideband PHY and MAC Standard, 2005.

[20] R. Llorente, T. Alves, M. Morant et al., "Ultra-wideband radio signals distribution in FTTH networks," IEEE Photonics Technology Letters, vol. 20, no. 11, pp. 945-947, 2008.

[21] M. Morant, J. Perez, R. Llorente, and J. Marti, “Transmission of 1.2 Gbit/s polarization-multiplexed UWB Signals in PON with $0.76 \mathrm{bit} / \mathrm{s} / \mathrm{Hz}$ spectral efficiency," in Proceedings of Optical Fiber Communication Conference (OFC '09), paper OTuJ6, March 2009.

[22] J. Perez, M. Morant, R. Llorente, and J. Martí, "Joint distribution of polarization-multiplexed UWB and WiMAX radio in PON," Journal of Lightwave Technology, vol. 27, no. 12, pp. 1912-1919, 2009.

[23] M. Morant, J. Pérez, and R. Llorente, "Effect of multi-channel MB-OFDM UWB radio-over-fiber transmission using polarization multiplexed distribution in FTTH networks," in Proceedings of the Access Networks and In-House Communications (ANIC '10), paper AThA6, June 2010.

[24] General Photonics, "Reset-free polarization stabilizer-PolaStay," http://www.generalphotonics.com/pdf/PolaStay.pdf.

[25] T. Pratt, B. Walkenhorst, and S. Nguyen, "Adaptive polarization transmission of OFDM signals in channels with polarization mode dispersion and polarization-dependent loss," IEEE Transactions on Wireless Communications, vol. 8, no. 7, pp. 3354-3359, 2009.

[26] M. T. Core, "Cross polarization interference cancellation for fiber optic systems," IEEE Journal of Lightwave Technology, vol. 24, no. 1, pp. 305-312, 2006.

[27] T. Alves and A. Cartaxo, "Performance degradation due to OFDM-UWB radio signal transmission along dispersive singlemode fiber," IEEE Photonics Technology Letters, vol. 21, no. 3, pp. 158-160, 2009. 

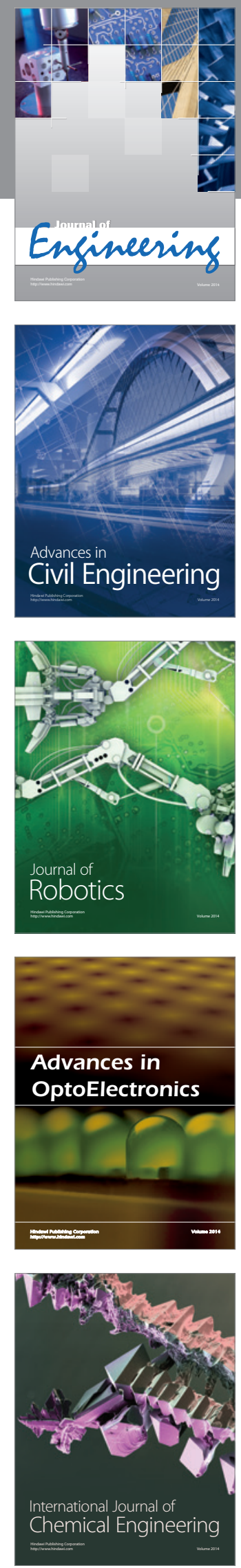

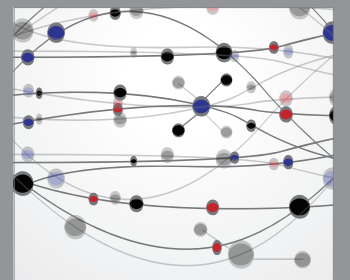

The Scientific World Journal
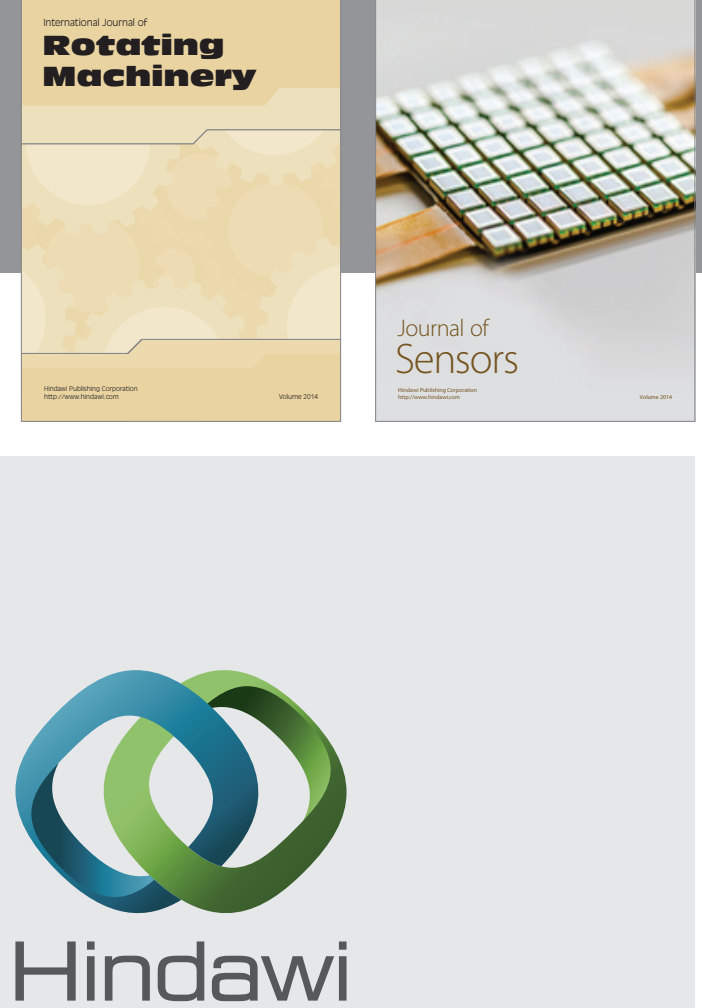

Submit your manuscripts at http://www.hindawi.com
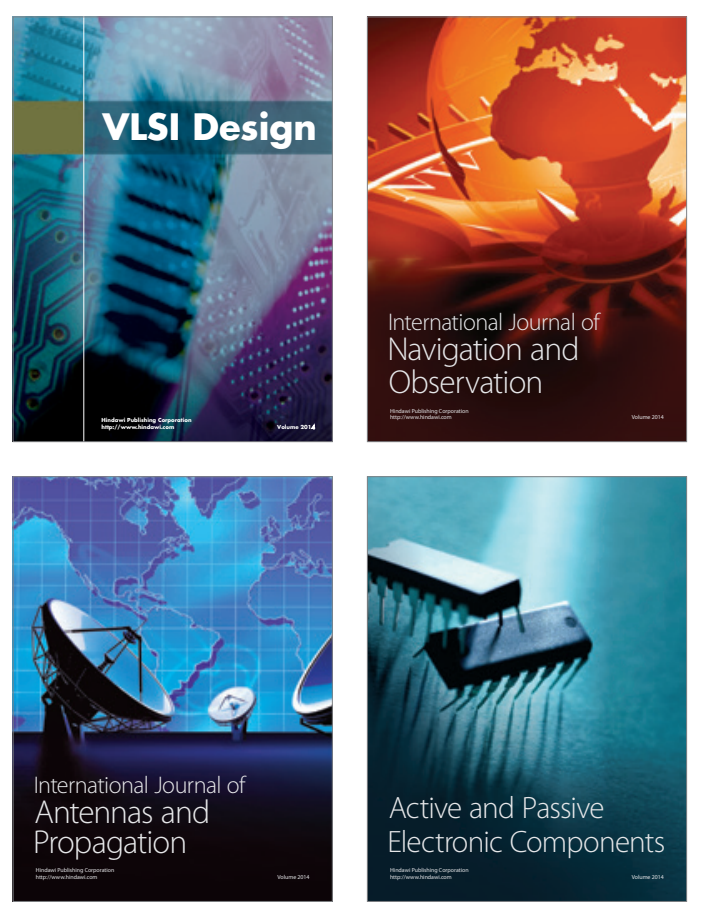
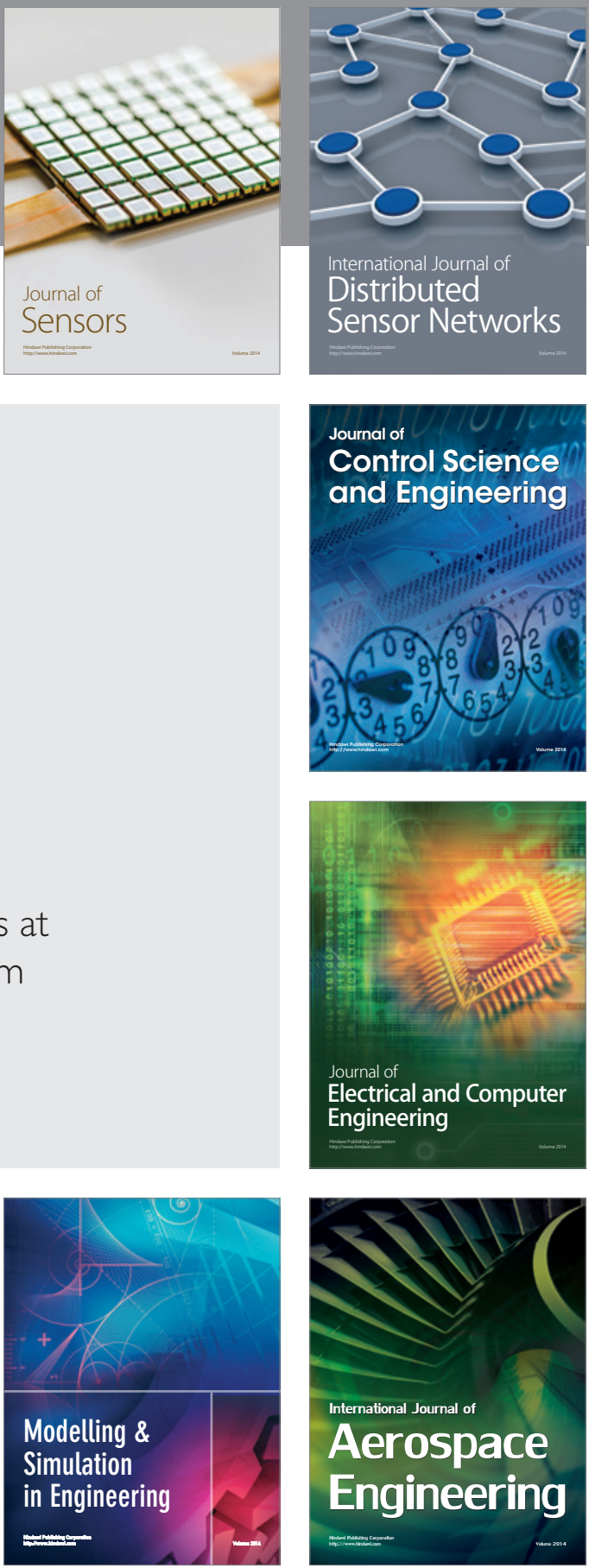

Journal of

Control Science

and Engineering
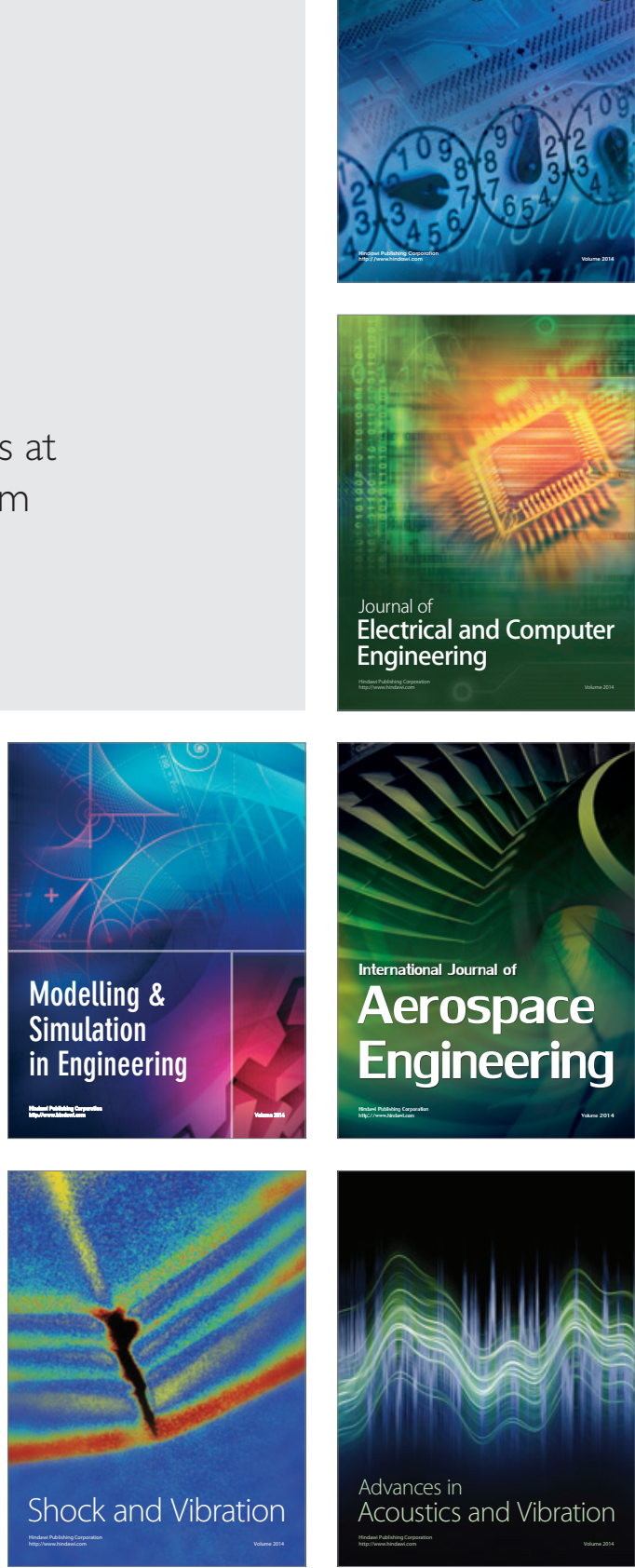